\title{
THE STATIONARY PROBABILITY DENSITY OF A CLASS OF BOUNDED MARKOV PROCESSES
}

\author{
MUHAMAD AZFAR RAMLI*** AND \\ GERARD LENG, ${ }^{* * * *}$ National University of Singapore
}

\begin{abstract}
In this paper we generalize a bounded Markov process, described by Stoyanov and Pacheco-González for a class of transition probability functions. A recursive integral equation for the probability density of these bounded Markov processes is derived and the stationary probability density is obtained by solving an equivalent differential equation. Examples of stationary densities for different transition probability functions are given and an application for designing a robotic coverage algorithm with specific emphasis on particular regions is discussed.
\end{abstract}

Keywords: Bounded Markov process; general state space; stationary density; Markov operator

2010 Mathematics Subject Classification: Primary 60J20

Secondary $60 \mathrm{~J} 05$

\section{Introduction}

Analyses of Markov processes in continuous state space are of much interest, especially in the fields of random particle dynamics and diffusion [1]. Markov processes are also found in studies of population theory [4], and even used in financial analyses of price fluctuations [9] and currency exchange rates [2]. Markov processes are also used to model the behaviour of swarm robots [7]. Owing to the physical nature of the variables involved in these processes, all these examples involve bounded state spaces.

In this paper we derive the stationary probability density function of a class of discrete-time Markov chains on the interval [0,1]. Consider the process described in [11], [12], and [13], where a discrete-time bounded Markov process $x_{n} \in[0,1], n=0,1,2, \ldots$, behaves as follows. Suppose that at time $n, x_{n} \in[0,1]$. At time $n+1$, the process has only two possible choices for transition, it either moves towards 0 with a probability $p\left(x_{n}\right)$ that is dependent on its current position or towards 1 with probability $1-p\left(x_{n}\right)$. Boundary considerations require that $p(0)=0$ and $p(1)=1$ in order that the process remains within $[0,1]$. After selecting a direction, the length of the change in position of the process within the selected interval is determined by a random variable $A \in[0,1]$. At time $n+1$, the process has therefore moved to

$$
x_{n+1}=x_{n}-A \times\left(x_{n}\right) \text { or } \quad x_{n+1}=x_{n}+A \times\left(1-x_{n}\right) .
$$

Figure 1 clearly illustrates this mechanism of random movement.

Received 28 January 2010; revision received 26 May 2010.

* Postal address: Cooperative Systems Lab E1-03-06, Department of Mechanical Engineering, National University of Singapore, 1 Engineering Drive 2, Singapore 117576.

** Email address: g0700822@nus.edu.sg

*** Email address: mpelsb@ nus.edu.sg 


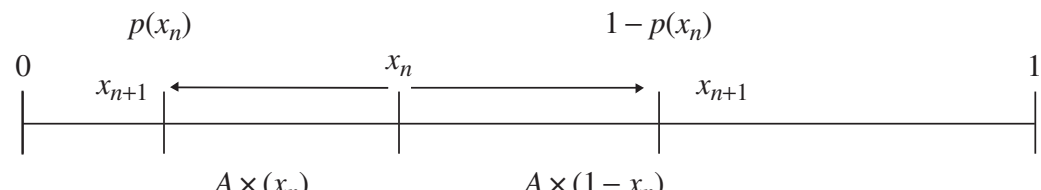

FiguRE 1: The bounded Markov process.

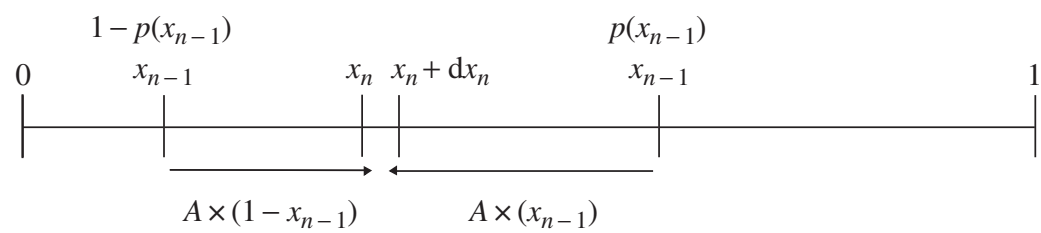

FIGURE 2: The transition process.

The solution of interest is the stationary probability density of the process after a large number of steps. In order to do this, we first use a backward equation to characterise the evolution of the probability density of the position of the process. Let $f_{n}(x)$ be the probability density function of the Markov process at time $n$. From Figure 2, it is clear that the probability that the process is found within the interval $\left(x_{n}, x_{n}+\mathrm{d} x_{n}\right)$ is

$$
\begin{aligned}
f_{n}\left(x_{n}\right) \mathrm{d} x_{n}= & \int_{0}^{x_{n}} f_{n-1}\left(x_{n-1}\right)\left(1-p\left(x_{n-1}\right)\right) \\
& \quad \times \mathrm{P}\left(\frac{x_{n}-x_{n-1}}{1-x_{n-1}}<A<\frac{x_{n}-x_{n-1}}{1-x_{n-1}}+\frac{\mathrm{d} x_{n}}{1-x_{n-1}}\right) \mathrm{d} x_{n-1} \\
& +\int_{x_{n}}^{1} f_{n-1}\left(x_{n-1}\right) p\left(x_{n-1}\right) \\
& \quad \times \mathrm{P}\left(\frac{x_{n-1}-x_{n}}{x_{n-1}}-\frac{\mathrm{d} x_{n}}{x_{n-1}}<A<\frac{x_{n-1}-x_{n}}{x_{n-1}}\right) \mathrm{d} x_{n-1} .
\end{aligned}
$$

In this paper we only study the case where $A \sim U(0,1)$, the standard uniform distribution, and, by replacing $x_{n-1}$ with dummy variables in the integrals, we obtain

$$
f_{n}\left(x_{n}\right)=\int_{0}^{x_{n}} f_{n-1}(u) \frac{1-p(u)}{1-u} \mathrm{~d} u+\int_{x_{n}}^{1} f_{n-1}(v) \frac{p(v)}{v} \mathrm{~d} v .
$$

The evolution of the probability density function of the process within the domain is now clearly defined.

We write (1) as the operator equation $f_{n}(x)=P f_{n-1}(x)$.

\section{Existence of the stationary density}

We need to understand the behaviour of the function $f_{n}$ when subjected to the operator $P$ as $n \rightarrow \infty$. For this purpose, we use the results from [6, Chapter 3] to prove the existence of the stationary density $f_{\infty}$. We first introduce a few basic definitions. 
Definition 1. The family of all possible real-valued functions $f: X \rightarrow \mathbb{R}$ satisfying

$$
\int_{X}|f(x)| \mathrm{d} x<\infty
$$

is known as the $L^{1}$ space.

Definition 2. Any linear operator $P: L_{1} \rightarrow L_{1}$ satisfying

(i) $P f \geq 0$ for $f \geq 0$ and $f \in L^{1}$;

(ii) $\|P f\|=\|f\|$ for all $f \geq 0$ and $f \in L^{1}$

is called a Markov operator.

Remark 1. Note that, for any $f \in L^{1}$, where $f$ is not given the abovementioned restrictions, the following inequality holds true for any valid Markov operator $P$ :

$$
\|P f\| \leq\|f\| .
$$

The proof of this result is trivial and can be found under Proposition 3.1.1 of [6, p. 39].

Proposition 1. The operator $P$ defined by (1) is a Markov operator.

Proof. Given that $f(u)$ is a valid probability density on [0,1], and since $u, v, 1-p(u), p(v) \in$ $[0,1]$, they are all greater than or equal to 0 , showing that $P f \geq 0$ and, thus, satisfying Definition 2(i).

Since $\|f\|=\int_{X}|f(x)| \mathrm{d} x=\int_{0}^{1} f(x) \mathrm{d} x=1$ and

$$
\begin{aligned}
\|P f\| & =\int_{X}\left|\int_{0}^{x} f(u) \frac{1-p(u)}{1-u} \mathrm{~d} u+\int_{x}^{1} f(v) \frac{p(v)}{v} \mathrm{~d} v\right| \mathrm{d} x \\
& =\int_{0}^{1} f(u) \frac{1-p(u)}{1-u} \int_{u}^{1} \mathrm{~d} x \mathrm{~d} u+\int_{0}^{1} f(v) \frac{p(v)}{v} \int_{0}^{v} \mathrm{~d} x \mathrm{~d} v \\
& =\int_{0}^{1} f(u) \mathrm{d} u \\
& =\|f\|,
\end{aligned}
$$

$P$ satisfies both conditions (i) and (ii) of Definition 1; therefore, we can conclude that $P$ is indeed a Markov operator.

Proposition 2. Define $f_{\infty}=P f_{\infty}$ to be the stationary density of the Markov operator $P$. Then, given any initial probability density function $f_{0} \in L^{1}, f_{0} \geq 0, \int_{X} f_{0} \mathrm{~d} x=1$,

$$
\lim _{n \rightarrow \infty} P^{n} f_{0}=f_{\infty}
$$

Proof. We can prove Proposition 2 by simply showing that $\lim _{n \rightarrow \infty}\left\|P^{n} f_{0}-f_{\infty}\right\| \rightarrow 0$ holds. We have

$$
\left\|P^{n} f_{0}-f_{\infty}\right\|=\left\|P^{n}\left(f_{0}-f_{\infty}\right)\right\|<\left\|P^{n-1}\left(f_{0}-f_{\infty}\right)\right\|=\left\|P^{n-1} f_{0}-f_{\infty}\right\| .
$$

The inequality is a direct result of (2). Note the strict inequality is used here because if both $f_{0}$ and $f_{\infty}$ are valid density functions then the function $f_{0}-f_{\infty}$ cannot satisfy condition (ii) of Definition 2. 
Now the norm of the difference between the $n$th iterate of $f_{0}$ and $f_{\infty}$ is always strictly less than the norm for the $(n-1)$ th iterate. This means that, as $n \rightarrow \infty,\left\|P^{n} f_{0}-f_{\infty}\right\| \rightarrow 0$, which completes the proof. Furthermore, we note from the above inequality that $P$ acts as a contraction mapping and, from Banach's fixed point theorem, we can conclude that $f_{\infty}$ is unique for all $f_{0} \in L^{1}$.

Different methods of proving the existence of stationary densities of other Markov processes can be found in [8, Chapter 10], which are also applicable to more general state spaces.

\section{General solution for the stationary probability density}

We now solve the stationary Markov operator equation $f_{\infty}=P f_{\infty}$. An explicit solution to this integral equation can be obtained by reducing it to an equivalent differential equation as follows.

Theorem 1. Let $f_{\infty}(x)$ be the stationary probability density of the Markov process described in Section 1. Then the explicit form of $f_{\infty}(x)$ for a fixed $p(x)$ is given by

$$
f_{\infty}(x)=C \exp \left(\int \frac{x-p(x)}{x(1-x)} \mathrm{d} x\right)
$$

where $C$ is a constant obtained by solving the probability density condition $\int_{0}^{1} f_{\infty}(x) \mathrm{d} x=1$.

Proof. From (1), substituting $n$ and $n-1$ with $\infty$, we obtain the integral equation

$$
f_{\infty}(x)=\int_{0}^{x} f_{\infty}(u) \frac{1-p(u)}{1-u} \mathrm{~d} u+\int_{x}^{1} f_{\infty}(v) \frac{p(v)}{v} \mathrm{~d} v .
$$

In order to solve for $f(x)$, we use Leibniz's integral rule [3] to convert the integral equation into an equivalent differential equation. The following equation shows the general form for Leibniz's integral rule:

$$
\frac{\mathrm{d}}{\mathrm{d} \alpha} \int_{a(\alpha)}^{b(\alpha)} g(x, \alpha) \mathrm{d} x=g(b, \alpha) \frac{\mathrm{d} b}{\mathrm{~d} \alpha}-g(a, \alpha) \frac{\mathrm{d} a}{\mathrm{~d} \alpha}+\int_{a(\alpha)}^{b(\alpha)} \frac{\partial}{\partial \alpha} g(x, \alpha) \mathrm{d} x .
$$

Remark 2. Even though Leibniz's rule requires that $g$ is a differentiable function, valid solutions for the stationary density can still be obtained if the function is discontinuous on at most a finite number of points in the domain.

By differentiating both sides of (4), and utilizing (5), we obtain the following simple separable differential equation, which is easily solved and whose solution is (3):

$$
f_{\infty}^{\prime}(x)=f_{\infty}(x) \frac{1-p(x)}{1-x}-f_{\infty}(x) \frac{p(x)}{x} .
$$

\subsection{Gradient of the stationary probability density}

Corollary 1. Differentiation of the stationary density function $f_{\infty}(x)$ gives

$$
f_{\infty}^{\prime}(x)=\frac{x-p(x)}{x(1-x)} C \exp \left(\int \frac{x-p(x)}{x(1-x)} \mathrm{d} x\right) .
$$

Remark 3. Note that, since $C>0$ and $\mathrm{e}^{x}>0$ for all $x$, the sign of the gradient of the distribution function within the interval $(0,1)$ is wholly dependent on the value $x-p(x)$. As such, if $p(x)<x$, we find that the distribution function is always increasing, vice versa for 
$p(x)>x$. In the next section we show that by selecting a suitable function $p(x)$ we can engineer different useful stationary densities.

Corollary 2. Owing to the restrictions on $p(x)$, the values of the gradient are bounded by

$$
0 \leq p(x) \leq 1, \quad-\frac{1}{x} \leq \frac{x-p(x)}{x(1-x)} \leq \frac{1}{1-x}, \quad-\frac{f_{\infty}(x)}{x} \leq f_{\infty}^{\prime}(x) \leq \frac{f_{\infty}(x)}{1-x} .
$$

Remark 4. Equation (7) specifies the allowable gradient of the class of probability density functions that can be generated by this Markov process.

\subsection{Examples of simple probability densities}

From the general solution found in (3), we can find a few simple examples of stationary probability densities that can be obtained by varying $p(x)$.

Example 1. Consider the case in which $p(x)=x$. The solution is simply the constant $f_{\infty}(x)=C$. Applying the distribution condition, it is trivial to show that $C=1$; thus, the obtained distribution is the standard uniform distribution along the interval $[0,1]$.

Remark 5. The case of Example 1 represents a centering condition on the Markov process, in which the probability that the process shifts to the right is directly proportional to the distance away from the right boundary, and similarly for the leftwards transition. As can be expected, the centering condition results in the process achieving a uniform distribution, and it is useful when wanting the process to have an equal chance of visiting all the sites within the domain.

Example 2. For a constant value $p(x)=p, x \in(0,1)$ and $p \in(0,1)$,

$$
f_{\infty}(x)=C x^{-p}(1-x)^{p-1},
$$

which is the beta distribution with parameters $\alpha=1-p, \beta=p$, and $C=1 / B(1-p, p)$, where $B(\alpha, \beta)$ denotes the beta function. Note that, since $p \in(0,1)$, the resulting distribution is always U-shaped. The resulting distribution will also skew towards the left or right side, depending on whether $p>1-p$ or $p<1-p$.

Remark 6. Example 2 demonstrates that when $p(x)$ is set to be a constant value, the distribution follows a generalized arcsine distribution [5, Chapter 25], causing the process to have an infinitely high probability density as it approaches the two boundary regions. This property is also commonly found in various diffusion processes, as well as bounded Brownian motions [10].

The solution found in (8) matches the solution of a special case of our problem first proved in [13] using a moment convergence theorem and matching the moments of the known beta distribution to the moments obtained by the Markov process. Our solution is however based on the direct solution of the integral equation of the probability density function.

Depending on the proposed application of this Markov process model, we may desire a moderately uniform distribution, in which case a constant $p(x)$ is not suitable, since the position of the particle has a tendency to stay at either boundary. Furthermore, we wish to influence the movement of the process such that it tends to visit certain states more than others. To this end, we use our knowledge of the gradient from Corollaries 1 and 2.

\section{Creating peak-valued probability density functions}

To exert a measure of control on the steady state movement of the random process by ensuring that some parts of the state space are visited more often than others, we will need to ensure that 

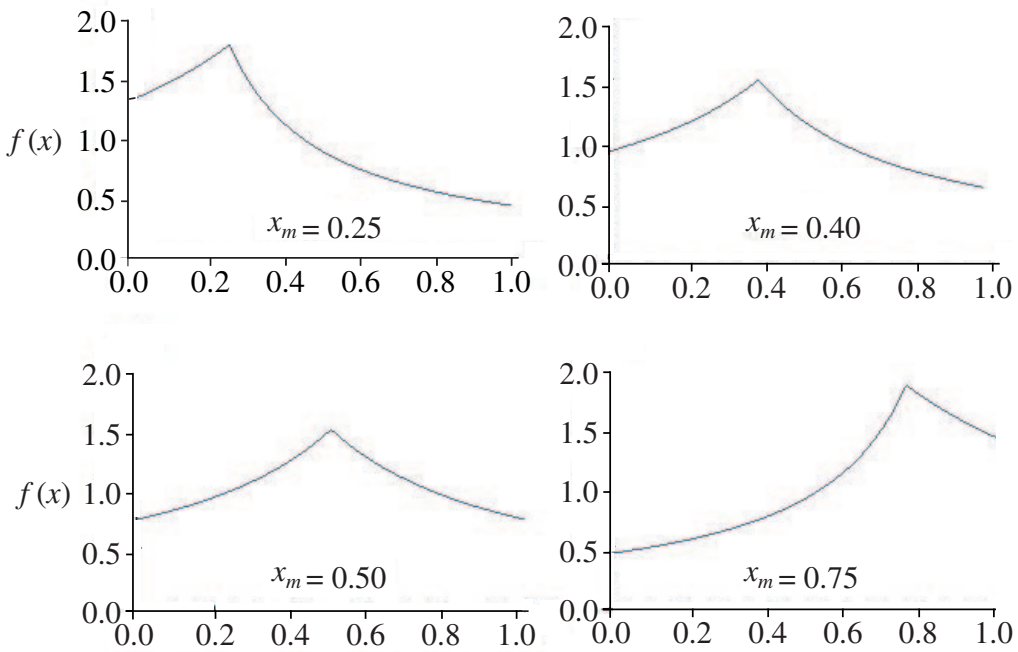

FIGURE 3: Peak-valued probability density functions.

we are able to engineer maximum or minimum points in the limiting distribution. In order to engineer a maximum point at $x_{m}$, we can deduce from (6) that we simply need to ensure that $p\left(x<x_{m}\right) \leq x$ and $p\left(x>x_{m}\right) \geq x$. This can easily be done using a simple step function for $p(x)$ :

$$
p(x)= \begin{cases}0, & 0 \leq x \leq x_{m} \\ 1, & x_{m}<x \leq 1\end{cases}
$$

The resulting piecewise stationary probability density is solved by simply substituting $p(x)$ for 0 and 1 in (8) for each subdomain:

$$
f(x)= \begin{cases}\frac{C_{1}}{1-x}, & 0 \leq x \leq x_{m}, \\ \frac{C_{2}}{x}, & x_{m}<x \leq 1 .\end{cases}
$$

Using the continuity criteria $f\left(x_{m}^{+}\right)=f\left(x_{m}^{-}\right)$as well as the standard probability density condition $\int_{0}^{1} f(x) \mathrm{d} x=1$, we can solve for the values of $C_{1}$ and $C_{2}$. We obtain

$$
C_{1}=\frac{1}{\ln \left[\left(1-x_{m}\right)^{-1} x_{m}^{x_{m} /\left(x_{m}-1\right)}\right]}, \quad C_{2}=\frac{1}{\ln \left[\left(1-x_{m}\right)^{\left(x_{m}-1\right) / x_{m}} x_{m}^{-1}\right]} .
$$

Figure 3 shows some examples of the peak-valued probability densities obtained for different values of $x_{m}$.

\section{Application to a two-dimensional random robotic coverage algorithm}

We show here how we apply the principles to a robot coverage movement algorithm used in a two-dimensional interior room. The objective of the robot is to ensure that the whole room is 
periodically covered by a sensor, with specific areas in the room given higher priority due to the proximity of important objects within the room. In order to reproduce the movement algorithm described by our bounded Markov process, the robot will be assumed to have localization capabilities, possibly via the use of distance sensors measuring the proximity of the nearby walls.

In our example the robot is given the task of covering a two-dimensional rectangular room of dimensions $X$ by $Y$ metres, and a single position of interest is found at $(0.2 X, 0.5 Y)$, which requires more visits than the rest of the room. We assume that the robot moves independently in the $X$ and $Y$ directions. Let the fraction of the distances between the robot and the wall over the length and breadth of the room at time $n$ be denoted by $X_{n}$ and $Y_{n}$, respectively, and let the robot move according to the following algorithm.

Algorithm 1. Step 1. Measure position $\left(X_{n}, Y_{n}\right)$ relative to the room walls.

Step 2. Select motion in the $X$ and $Y$ directions according to the following rules.

- if $0<X_{n}<0.2$ then select the positive $X$ direction else select the negative $X$ direction;

- if $0<Y_{n}<0.5$ then select the positive $Y$ direction else select the negative $Y$ direction.

Step 3. Select and travel a random distance as a fraction of the available space as follows:

$$
\text { distance travelled }=\left\{\begin{array}{l}
\text { positive } X \text { direction: } A_{x} \times\left(1-X_{n}\right), \\
\text { negative } X \text { direction: } A_{x} \times\left(X_{n}\right), \\
\text { positive } Y \text { direction: } A_{y} \times\left(1-Y_{n}\right), \\
\text { negative } Y \text { direction: } A_{y} \times\left(Y_{n}\right),
\end{array}\right.
$$

where $A_{x}, A_{y} \sim U(0,1)$.

Step 4. Loop back to step 1 .

A simulation showing the number of times the robot visited each position within the room after 10 million time steps was conducted using MATHEMATICA ${ }^{\circledR}$ and the resulting probability density plotted in Figure 4 . Figure 4 shows that the desired number of visits to the area is significantly higher at the point of interest, $(0.2 \mathrm{X}, 0.5 \mathrm{Y})$, as required.

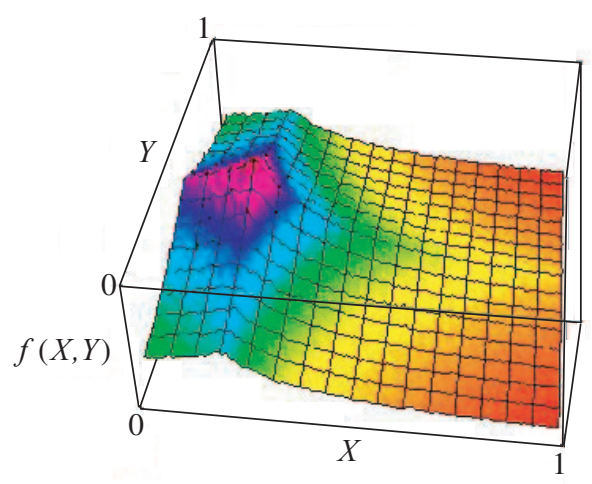

FIGURE 4: Probability density plot of the two-dimensional random coverage algorithm. 


\section{Conclusion}

We derived a recursive integral equation for the evolution of the probability density of a class of bounded Markov processes. By solving an equivalent differential equation, we obtained the general solution of the stationary probability density of the given Markov process for different transition probability functions. We also showed that when a step function is used for the transition probability function, it results in a peak-valued stationary density. Finally, we showed how this allows us to design a suitable algorithm for a two-dimensional random area coverage problem within a simple rectangular room.

\section{References}

[1] Bargiel, M. and Tory, E. M. (2007). A five parameter Markov model for simulating the paths of sedimenting particles. Appl. Math. Modelling 31, 2080-2094.

[2] Farahpour, F. et al. (2007). A Langevin equation for the rates of currency exchange based on the Markov analysis. Physica A 385, 601-608.

[3] Flanders, H. (1973). Differentiation under the integral sign. Amer. Math. Monthly 80, 615-627.

[4] Hernández-Suárez, C. M. and Castillo-Chavez, C. (1999). A basic result on the integral for birth-death Markov processes. Math. Biosci. 161, 95-104.

[5] Johnson, N. L., Kotz, S. and Balakrishnan, N. (1995). Continuous Univariate Distributions, Vol. 2, 2nd edn. John Wiley, New York.

[6] Lasota, A. And Mackey, M. C. (1994). Chaos, Fractals, and Noise, 2nd edn. Springer, New York.

[7] Lerman, K., Martinoli, A. And Galstyan, A. (2005). A review of probabilistic macroscopic models for swarm robotic systems. In Swarm Robotics Workshop: State-of-the-art Survey (Lecture Notes Comput. Sci. 3342), eds E. Sahin and W. Spears, Springer, Berlin, pp. 143-152.

[8] Meyn, S. P. And Tweedie, R. L. (1993). Markov Chains and Stochastic Stability. Springer, London.

[9] NiELSEN, C. K. (2009). Non-stationary, stable Markov processes on a continuous state space. Econom. Theory 40, 473-496.

[10] Nikitin, Y. and Orzinger, E. (2000). The intermediate arc-sine law. Statist. Prob. Lett. 49, 119-125.

[11] Pacheco-González, C. G. (2009). Ergodicity of a bounded Markov chain with attractiveness towards the centre. Statist. Prob. Lett. 79, 2177-2181.

[12] Pacheco-González, C. G. and Stoyanov, J. (2008). A class of Markov chains with beta ergodic distributions. Math. Sci. 33, 110-119.

[13] Stoyanov, J. And Pirinsky, C. (2000). Random motions, classes of ergodic Markov chains and beta distributions. Statist. Prob. Lett. 50, 293-304. 